\title{
Du corps au cancer : la construction métaphorique de l'ennemi intérieur dans le discours militaire pendant la Guerre Froide. Partie 1
}

\section{Gabriel Périès}

\section{(2) OpenEdition \\ Journals}

\section{Édition électronique}

URL : http://journals.openedition.org/conflits/864

DOI : $10.4000 /$ conflits.864

ISSN : $1777-5345$

Éditeur :

CCLS - Centre d'études sur les conflits lilberté et sécurité, L'Harmattan

Édition imprimée

Date de publication : 1 septembre 2001

ISBN : 2-7475-1118-9

ISSN : $1157-996 \mathrm{X}$

\section{Référence électronique}

Gabriel Périès, « Du corps au cancer : la construction métaphorique de l'ennemi intérieur dans le discours militaire pendant la Guerre Froide. Partie $1 »$, Cultures \& Conflits [En ligne], 43 | automne 2001, mis en ligne le 28 février 2003, consulté le 30 mars 2021. URL : http://journals.openedition.org/ conflits/864 ; DOI : https://doi.org/10.4000/conflits.864

Ce document a été généré automatiquement le 30 mars 2021.

Creative Commons License 


\title{
Du corps au cancer : la construction métaphorique de l'ennemi intérieur dans le discours militaire pendant la Guerre Froide. Partie 1
}

\author{
Gabriel Périès
}

L'objet de notre travail porte sur la pratique de la dénomination de l'ennemi dans le cadre des doctrines militaires qui émergent à l'occasion de la Guerre Froide. Une de leur principale caractéristique a été de penser la gestion efficace de l'action coactive, coercitive et d'annihilation, non pas sur un ennemi provenant uniquement de l'extérieur des frontières, mais sur un ennemi agissant depuis l'intérieur du pays, émergeant du territoire national lui-même ou de l'Empire. Les doctrines militaires ont pris alors, en France, des appellations particulières: doctrine de la "guerre révolutionnaire " $(\mathrm{DGR})^{1}$, guerre subversive ${ }^{2}$ ou stratégie indirecte ${ }^{3}$. Sans parler, audelà des codifications doctrinales, des expériences grecques, argentines, turques... en la matière qui, très souvent sont venues influencer telle ou telle codification doctrinale en l'alimentant de leurs expérimentations.

Dans ce contexte doctrinal spécifique, une permanence langagière: la construction institutionnelle de l'ennemi de l'intérieur à travers des métaphores de nature médicochirurgicale (MMC). A titre d'illustration, citons un extrait du rapport Enseignement de la guerre d'Indochine, édité en 1955 par le Commandement en chef en Extrême-Orient, dans lequel on peut lire : «A l'actif du bilan comptent seulement les actions de 'contrôle en surface' qui visent àextirper ${ }^{4} \mathrm{~d}^{\prime} u n e$ région les rebelles qui $s^{\prime} y$ dissimulent. Cette chirurgie, fondée sur le diagnostic des localités les plus contaminées et de villages encore relativement sains, doit amener l'ablation des tissus gangrenés et ouvrir la voie à cette convalescence, qui sera la pacification proprement dite $»^{5}$. Au-delà de ces écrits de synthèse technique, internes à l'armée, nous trouvons aussi l'utilisation de la MMC dans des ouvrages à vocation plus théorique traitant, par exemple, de la stratégie indirecte dans le contexte de la Guerre Froide. Ainsi, le général Beaufre a-t-il recours à 
cette forme de trope pour libeller un certain nombre de chapitres et de sous-chapitres de son livre Stratégie de l'action ${ }^{6}$; Action et diagnostic politique » (p.31); «Dissection du concept d'action" (p.53); "Anatomie de la force: le niveau d'action» (p.81); «Inventaire politique et diagnostic politique» (p.82); "Inventaire stratégique et diagnostic stratégique » (p.86).

On relèvera également la présence de $\mathrm{MMC}$ dans des écrits de militaires étrangers ayant reçu l'enseignement de la DGR. La MMC est ainsi utilisée dans le discours des militaires argentins, en 1977, lorsque, définissant «l'étape fondatrice » du « Processus de réorganisation nationale $»^{7}$, ils soulignent qu'il convient de "gagner une guerre cruelle et d'une énorme complexité, pendant laquelle, à l'activité créatrice se juxtapose, simultanément, l'action chirurgicale nécessaire à l'ablation de l'omnipotent cancer de la subversion communiste $»^{8}$.

Dans un tel contexte énonciatif, que devient l'ennemi, quelles figures prend-il ? Et audelà, quelles représentations du professionnel de la violence légitime de tels mécanismes dénominatifs structurent-ils ? Cette pratique langagière est-elle neutre sur le plan du comportement du militaire, qu'indique-t-elle par rapport à ses relations avec le pouvoir civilo-politique?

Pour répondre à ces interrogations, on portera l'attention sur trois aspects spécifiques de la question. Premièrement ${ }^{9}$, on procédera à une description détaillée des principales formes performantes de l'emploi de la MMC aussi bien d'un point de vue rhétorique qu'au sein de l'économie générale d'un discours spécifique qui se donne volontairement pour objectif son emploi. A cette occasion, on analysera les réseaux métaphoriques ${ }^{10}$ présents dans un texte produit par un officier français au cours de la guerre d'Algérie ${ }^{11}$, pour relever que l'usage de la MMC s'insère dans le discours militaire comme un vecteur des représentations sociales et politiques d'au moins une partie de l'armée française. Mais les représentations de l'ennemi conditionnent également celle de l'ami. Dans un second temps, on observera comment le recours à la MMC induit une schématisation organiciste des représentations corporelles de l'ami comme de l'ennemi, qu'il militarise. Enfin, dans un troisième temps, toujours en suivant les conditions d'emploi de la MMC et des représentations organicistes, on observera comment, pendant la Guerre Froide, le discours institutionnel militaire offre une véritable tératologie idéologique qui va depuis la construction de chimères sociales jusqu'à l'affirmation d'une organisation contre-révolutionnaire dans l'optique de tuer le microbe et ensuite de cicatriser les plaies de la Nation ${ }^{12}$.

Typologie indicative et étude de cas de l'utilisation de la MMC dans le discours militaire Etablir une typologie, toute indicative, de l'utilisation de la MMC dans le discours militaire revient à détecter, puis à décrire, les formes et les fonctions qu'elle remplit au sein d'une argumentation destinée à transmettre des connaissances et/ou à convaincre un auditoire, ou un lecteur, de la pertinence d'un discours ${ }^{13}$. Il s'agit donc de décrire les emplois performants de cet outil de rhétorique lorsqu'il est placé à l'intérieur d'un schéma argumentatif, eu égard à un autre outil, celui de l'analogie, qu'il est sensé porter ${ }^{14}$. Par la suite on dégagera du texte Guerre révolutionnaire et médecine moderne les principaux réseaux métaphoriques qui structurent les représentations du militaire et de son action comme celles de l'ennemi.

Analogies et métaphores relevant de l'emploi de la MMC : une approche rhétorique

Dans cette optique, nous avons isolé trois types de relation entre la MMC et l'analogie, ce qui implique trois manières différentes d'organiser un schéma argumentatif. Les 
deux premiers ont comme substrat un texte écrit, le troisième repose sur un support matériel d'une autre nature: une représentation cartographiée, géographique, de l'ennemi et sa désignation lexicalisée.

Premier type. Les MMC, dans leur enchainement, portent une analogie introductive contenant des sèmes ${ }^{15}$ à référentiel médico-chirurgical

"Cette guerre froide est à la guerre chaude ce que la médecineest à la chirurgie. Aux opérations sanglantes de la guerre chaude se substituent les 'infections'qui ne sont pas moins meurtrières, mais plus insidieuses. Contre ces infections, la méthode chirurgicale est rarement efficace: il faut procéder à des vaccinations préventives ou à des contreinfections, et il faut prendre la maladie dès le début. Dans cette guerre larvée où les infections psychologiques ressemblent à celles de la guerre biologique, il est très difficile de contrôler les phénomènes une fois déclenchés: l'Allemagne a succombé en 1918 en grande partie à cause du retour du virus bolchevique qu'elle avait contribué à semer en Russie un an plus tôt ; le prurit de décolonisation sur lequel les Soviétiques avaient misé dès 1921 a parfois dépassé les prévisions de l'URSS et lui a posé en Afrique des problèmes auxquels elle n'était pas prête à répondre. Cette guerre médicale est très différente de nos habitudes, malgré son emploi millénaire ${ }^{16}$.

Dans ce premier exemple, le schéma argumentatif se développe en deux étapes: dans un premier temps, le locuteur fixe le contexte énonciatif [lignes 1 à 5] en ouvrant son propos par l'établissement d'une analogie à vocation généralisatrice; dans une seconde phase, il explicite la partie précédente. Il l'illustre par des exemples historiques assez précis et des comparaisons à vocation analogique. La dernière MMC de l'extrait ferme l'argumentation et accrédite l'affirmation analogique de départ.

Dans la première partie, l'enchaînement des métaphores est incorporé à la suite d'une analogie contenant des sèmes médico-chirurgicaux. L'utilisation des MMC consécutives se trouve autorisée tant par la présence de ces mots que par la polysémie du terme opérations [2], qui appartient aussi bien au lexique de l'art militaire qu'à celui de la médecine ou de la chirurgie. Cette ambiguïté fait office de maillon entre l'analogie et les métaphores qui suivent. Cela dit, l'auteur avance prudemment. Le recours aux guillemets, puis leur suppression, pour le même mot : infections [3 et 4$]$ est à signaler. Dans un premier temps, leur emploi renforce la présence de la métaphore qui accrédite la pertinence de l'analogie par un phénomène de distanciation; tandis que leur élimination rend fluide l'enchaînement des MMC qui suivent. Celles-ci - vaccinations préventives et contre-infections - possèdent une fonction particulière : elles se réfèrent à des types d'action militaire spécifique qui sont évoqués, alors, sur le mode elliptique ${ }^{17}$. La compréhension pleine de ces termes est impossible pour le non-professionnel. La MMC porte de la sorte un jargon de métier propre aux pratiques de la guerre froide qu'analyse l'énonciateur; cette fonction elliptique de la MMC se retrouve à la fin de la première partie du paragraphe, dans laquelle la métaphore désigne, par une catégorie large, la maladie, l'ennemi qu'il faut combattre sans pour autant qu'il en soit donné une définition précise. On sait tout de même qu'il s'agit d'une pathologie.

La suite de l'extrait est ouverte par une comparaison entre les infections psychologiques et la guerre biologique, ce qui relance l'argumentation. L'adjectif psychologique constitue une approche évocatrice d'un double phénomène, celui de l'idéologie, réduite à une variable en général béhavioriste ${ }^{18}$, et celui de sa diffusion par la propagande ${ }^{19}$. L'expression guerre biologique renforce la métaphore antérieure en lui conférant un contenu pratique et en la liant à un type concret de guerre, bien connu par le lecteur 
militaire ou spécialisé en la matière: celui dans lequel on utilise des armes bactériologiques, par exemple. Les deux métaphores suivantes, celles de virus bolchevique et de prurit de décolonisation, désignent l'ennemi lui-même et les «symptômes » qu'il suscite sur le mode de l'adjectivation ou de la complémentarité. L'ellipse, induite plus haut dans l'extrait par le terme maladie se trouve ainsi levée; l'ennemi est maintenant clairement désigné. Enfin, la métaphore de guerre médicale[16] accrédite l'analogie introductive et affirme, par ce biais, les orientations que l'auteur entend donner aux missions de l'armée.

Deuxième type. Les MMC du schéma argumentatif fonctionnent majoritairement comme des analogies condensées ${ }^{20}$. "L'exposé de ces actions serait trop concis si l'on ne mettait pas l'accent sur les facteurs suivants : la nécessité de le commencer par une brève analyse des origines dukyste de la subversion, extirpépar les forces armées avec rapidité et efficacité opérationnelle, face à la menace tangible que la maladie ne $s^{\prime}$ aggraveet ne se transforme enun incurable cancer $»^{21}$.

Dans le schéma argumentatif de ce paragraphe, il n'y a pas d'analogie formellement reconnaissable, ni de comparaison. Pourtant, la première MMC : kyste de la subversion, porte en elle une analogie condensée. Contrairement au cas précédent, elle n'intervient pas à la suite d'une introduction qui campe des expressions appartenant au référentiel médico-chirurgical. L'emploi métaphorique est directement intégré au discours et n'a pas besoin de se justifier. Il constitue une marque énonciative somme toute triviale, propre à la culture du locuteur. Composée de deux sèmes relevant de deux référentiels différents, cette métaphore constitue un amalgame qui se trouve renforcé par le participe extirpé qui valorise le trait kyste.

La MMC qui repose sur le syntagme la maladie ne s'aggrave désigne, d'une manière elliptique, l'ennemi et la situation dynamique générale qu'il suscite. Par la prévision de la dégénérescence du kyste en un incurable cancer, cette dernière métaphore accrédite l'analogie condensée du début du paragraphe, et vient entériner ce que l'auteur entend évoquer dans son ouvrage: la chronique de l'exercice efficace, d'une opération de contre-guérilla menée à bien $^{22}$; tout comme elle affirme une compétence professionnelle spécifique : un savoir prédictif qui se mue en autorité par compétence.

Troisième type. La MMC repose sur une analogie instaurée par une représentation graphique $^{23}$

Figure 1

Représentation métaphorique de l'ennemi : la carteRubéole

Ce cas est intéressant car il visualise la guérilla en Indochine ${ }^{24}$ comme Rubéole. Il convient de préciser que la MMC se situe ici dans un cadre spécifiquement militaire, puisque ce genre de carte n'est réservé, sous couvert du secret, qu'aux seuls professionnels. Nous sommes, comme dans le cas décrit précédemment, dans la situation de l'ellipse qui marque l'existence d'un jargon de métier. Dans ce cas, Rubéole est un terme technique qui désigne, à un moment précis et dans une situation géographique bien déterminée, l'implantation des «foyers »- foyers infectieux ? - de guérillas au Tonkin, au mois de mai 1954.

En résumé, deux observations se dégagent de l'utilisation rhétorique de la MMC dans le discours militaire. La première est qu'elle est performante sur le plan du schéma argumentatif. Elle porte le sens sans briser l'intelligibilité du discours. La deuxième est que l'emploi de cette catégorie de tropes couvre non seulement l'existence d'un jargon 
technique, mais exprime aussi un accomplissement pratique. Ce dernier point, qui met l'accent sur l'expression métaphorique de l'activité instrumentale propre au champ militaire, permet de mettre en perspective l'emploi de ce jargon médico-chirurgical en le replaçant dans son contexte socio-historique. Il devient alors possible de s'interroger sur le sens du choix opéré par le militaire dans l'utilisation de la MMC et, ainsi, de montrer que ce choix exprime des représentations qu'il se fait de son propre rôle et de la société dans laquelle il agit.

"Guerre révolutionnaire et médecine moderne » : étude de cas

Publié au mois de mai 1957, dans la Revue militaire d'information ${ }^{25}$, l'article qui nous occupe porte explicitement, dans son titre, une comparaison : guerre révolutionnaire et médecine moderne. Il exprime l'opinion anonyme d'un professionnel de l'art militaire sur le conflit algérien et ce, en pleine bataille d'Alger. Texte doublement circonstancié puisque, non seulement il s'insère dans le corpus doctrinal de la «guerre révolutionnaire" dont la revue mentionnée plus haut est l'un des principaux vecteurs ${ }^{26}$, mais aussi parce qu'il puise sa substance dans le contexte de terrorisme et de contre-terrorisme, d'action psychologique et d'encadrement des populations, si caractéristiques de l'époque.

C'est que dans le conflit colonial en période de guerre froide, la relation Ami-Ennemi qui a servi jusque-là de fondement à toute vision fonctionnelle sur le plan militaire, se transforme radicalement. A l'époque de la conquête de l'Empire, l'armée se trouvait, presque toujours, placée en posture offensive et la société du conquis était, par ethnocentrisme ${ }^{27}$, niée. Lors des conflits de l'après-Seconde Guerre Mondiale, le militaire est en position défensive et est obligé de tenir compte de l'existence de la société coloniale. Dans cette optique, il doit protéger tout autant le cadre territorial de la colonie que l'ordre social fortement contesté par la rébellion.

Le concept même d'Ami-Ennemi subit dès lors un déplacement et est dorénavant réservé à la lutte idéologique internationale où s'affrontent Monde Libre et Communisme, tandis que, sur le plan opérationnel, local, il prend une forme ternaire : Ami-Corps social-Ennemi. En effet, l'antagonisme guerrier se manifeste sur le terrain, au-delà de l'affrontement binaire de deux groupes armés: l'enjeu est coactif et coercitif. La guerre ne repose plus sur l'anéantissement de l'ennemi. Son but est le contrôle du corps social colonial ou sa destruction; et il s'agit, pour l'armée, de le préserver des menaces destructrices que fait peser sur lui l'insurrection.

Dans le texte en question, cinq réseaux de MMC peuvent être déterminés. Le premier a pour référent les armées, ou le on à vocation de nous; le second métaphorise les types d'action des forces armées ; le troisième est relatif à la désignation du corps social et à la société ; en ce qui concerne le quatrième réseau, il s'agit des référents désignant l'ennemi et leurs équivalents métphoriques; enfin, les syntagmes comprenant le mot santé ont été placés dans leur contexte argumentatif : la transformation de la relation Ami-Ennemi en Ami-Corps social-Ennemi.

Tableau 1 : réseau métaphorisant les arméesRéférents Réseau des équivalents sémantiques - MMC

«les armées»

ou « on » Bactéricides ; agents destructeurs ; médecin(s); praticien ; confrères ; agents spécifiques extérieurs; médecin des sociétés ; médecin de familles.

Tableau 2 : réseau métaphorisant les actions des arméesRéférents Réseau des équivalents sémantiques - MMC 
[actions des

armées] Dose ; cure ; arsenic; bismuth; intoxiqué(r); pénicilline ; diagnostic ; thérapeutique; consultation ;

Bilan physiologique ; radiographie ; mesures électriques ; analyses ;

Dosages, progrès sanitaire ; hormones ; vitamines ; « lyse » .

Tableau 3 : réseau métaphorisant lecorpssocial ou la « société »

Référents Réseau des équivalents sémantiques - MMC « corps social »

"Société » Organisme malade ; organisme ; affections ; lésions ; le malade ; membre atrophié ; s'alite $(\mathrm{r})$ la fièvre monte ; la rechute; l'état général du patient ; réaction organique; son malaise; les points faibles de l'état général; l'organisme; luttera; terrain biologiquement actif ; corps social ; les cellules ; corps sain.

Tableau 4 : réseau métaphorisant l'ennemiRéférents Réseau des équivalents sémantiques - MMC

« le Viet » Microbe meurtrier ; l'infection ;

Microbe pathogène « le Mau-Mau » Germes nuisibles ; le mal ; gangrène(r) ;

« Rubéole » « les rebelles » Le microbe mort ou enkysté ; le microbe adulte ; l'affection rebelle ; les facteurs pernicieux ;

«phagocytés» «(Moscou, Canton, Le Caire...)» Des bouillons de culture neutres ou favorables

à sa croissance (ne concerne directement que ce référent)

Le cinquième réseau métaphorique répond à une utilisation logico-argumentative spécifique du terme santé dans le cadre de la relation Ami-Ennemi, puis dans la cadre Ami-Corpssocial-Ennemi :

1. L'auteur fait un constat, analysant la guerre d'Indochine :

- Ami : « on »« interdit la vie au nom de la santé ».

- Ennemi : « la population (...) refuse cette santé ».

2. L'auteur propose une solution au conflit algérien par un retour à la tradition des pratiques de la Coloniale :

- Le Corps social, aidé par l'armée, produit « l'auto-défense de la santé ».

- L'Ennemi devient : « les éléments réfractaires à l'ordre de la santé ».

- L'Ami est : la Coloniale, incarnée par "Galliéni et Lyautey » : « Ils avaient découvert (...) le moyen de déclencher les processus de santé » .

L'analyse des cinq réseaux permet de dégager un certain nombre de remarques. Grâce à l'ambiguïté de l'expression corps social, le lecteur est renvoyé à une des représentations totalisantes et anciennes de la société et de l'Etat: celle de l'organicisme. La compréhension de cette représentation souligne la profonde assimilation culturelle de ce que l'on peut appeler une métaphore endormie ${ }^{28}$, et des évocations qu'elle réveille, tant chez l'énonciateur que chez le récepteur. La pertinence du discours repose sur l'acceptabilité de l'expression «corps social », relevant du sens commun. Par le biais de 
la perspective biologique que propose l'auteur du texte, les métaphores de la profession militaire, celles des spécialistes, du praticien, des confrères spécialisés..., est projeté sur le métier des armes le statut valorisant du scientifique, et du scientifique qui guérit parce qu'il possède un savoir - sans doute celui de la Coloniale. De plus, cette valorisation, par l'analogie que porte la MMC, place d'emblée l'acte guerrier sur le terrain de la neutralité axiologique attribuée aux sciences et évoque une déontologie qui valorise aussi bien les techniques guerrières efficaces qu'un savoir-faire sectoriel.

On constate également que le réseau qui concerne les techniques ne possède pas de référents ; volonté d'exprimer un jargon de métier sous couvert de l'ellipse induite par la métaphore? Il n'en reste pas moins que l'expression pseudo-métaphorique de mesures électriques ${ }^{29}$ peut être considérée comme une allusion à la pratique de l'interrogatoire nécessaire à la recherche du renseignement et au maintien de l'ordre propres aux « guerres révolutionnaires $»^{30}$.

Par contre, à la différence de l'armée qui se trouve valorisée, l'ennemi est assimilé à l'image péjorative du microbe. Il est sans aucune légitimité, puisqu'il provient de l'extérieur du corps social, il est issu du bouillon de culture " Moscou, Canton, Le Caire... », et sa seule action consiste à gangrener le malade, idéalement sain. A ce propos, le corps malade de celui-ci est passif : alité, rongé par la fièvre, il ne peut présenter que des lésions et est sujet à des rechutes, même si, dans un futur hypothétique, il luttera, aidé en cela par le médecin des sociétés, forme actualisée et adaptée à la situation nouvelle du médecin officiel des épidémies.

Cette dernière expression donne tout son sens au syntagme ordre de la santé, noyau métaphorique de la légitimation de l'interventionnisme militaire dans le corps social, affirmant de la sorte la licéité d'un ordre social militarisé qui fait pièce aux situations historiques de "pathologie politique ». Ainsi, le texte de 1957, Guerre révolutionnaire et médecine moderne semble annoncer une aptitude professionnelle nouvelle, faite pour répondre aux situations de guerre dont l'enjeu est surtout social et politique.

Cependant, au-delà de ce qui semblerait être un positionnement interne au champ militaire, c'est le poids de la MMC qui parait déterminant. Instrument rhétorique et puissant vecteur des représentations que projette le professionnel de la violence légitime sur lui et les autres, elle devient l'outil d'expression d'une conviction profondément ancrée et partagée. Et même si le militaire ne maîtrise pas, en scientifique, le vocabulaire, le lexique de la médecine ou de la chirurgie ou de la biologie..., il parle en savant, de quelque chose qu'il ne peut aborder qu'indirectement d'une manière autonome: le pouvoir. La MMC, par sa présence dans le discours militaire de la Guerre Froide, devient un véritable marqueur d'un glissement au sein du champ en question - ou de certains de ses secteurs - de l'éthique de la responsabilité vers l'éthique de la conviction. Reste à détecter puis à vérifier tant le mode d'élaboration que la nature des constructions sociales et politiques effectivement présentes dans le cadre de cet énoncé.

C'est en allant plus loin dans la détermination discursive des représentations liées aux thématiques de la santé et du corps dans le cadre de la gestion de la violence institutionnelle que nous allons saisir le complémentaire métaphorique dans lequel s'insère l'emploi de la MMC : à savoir les conditions énonciatives qui structurent, dans la cadre militaire, le thème du corps aussi bien celui de l'ami que celui de l'ennemi.

Les corps militarisés : métaphore de la Guerre Froide 
L'espace doctrinal français s'est nourri pendant la Guerre Froide d'un ensemble d'expériences et de positionnements d'autres armées dans le domaine de la lutte contre l'ennemi de l'intérieur, de la lutte contre la guérilla dans un contexte au sein duquel des jeux de représentations, d'auto-représentations et de représentations de l'ennemi ont pris des formes complémentaires. Ces jeux ont également abouti à des prises de position très agressives vis-à-vis du politique qui ont mené l'armée française, comme beaucoup d'autres après elle, à prendre des postures prétoriennes ou à rendre particulièrement labile la séparation institutionnelle entre le pouvoir militaire et l'autorité du politique, du civil.

Un lien discursif unit ces différentes postures et présente l'autre aspect de l'emploi de la MMC : le recours à une représentation organiciste de soi-même, de la société et de l'ennemi. Ce recours repose pour l'essentiel, sur un certain discours du corps, des corps, aussi bien du sien propre, que de celui de l'officier, que de l'organisation ennemie, que de celui des chimères sociales et idéologiques qui lui sont imputées.

Des métaphores organicistes et médicales complémentaires

En 1955, le centre de documentation de l'Ecole Supérieure de Guerre distribue aux stagiaires, sous la forme d'un seul document, deux textes, le premier est intitulé Exploitons la Révolution de l'Art militaireet est signé du commandant Cushman ${ }^{31}$ de l'Armée des Etats-Unis. Le thème essentiel est la modernisation des structures et de la technique militaire à la suite de la Seconde Guerre Mondiale. Le second article, "Les événements de Malaisie : une guerre froide » paru sous la signature du Général Brazer$\mathrm{Creagh}^{32}$, retrace les expériences britanniques en Malaisie, en 1954. Rien à première vue n'assure une unité thématique de ces deux textes, et pourtant l'ESG a cru bon les réunir. Un des fils directeurs est justement l'emploi rhétorique des métaphores organicistes et médico-chirurgicales qui vont mettre au centre du dispositif argumentatif une certaine représentation de l'armée, de l'ennemi et du spécialiste de la question politico-militaire :

Texte $n^{\circ} 1$ :

[a]« L'Armée est une chose vivante. Elle a une âme. Elle grandit et dépérit, elle croît et diminue. Comme toutes les choses vivantes, elle obéit à une des lois fondamentales de la nature, la loi de survivance du plus adapté. Notre Armée de Terre se trouve confrontée au problème que rencontrent toutes les choses vivantes depuis le commencement des temps, le problème de l'évolution, le problème de sa propre adaptation à des circonstances changeantes. Comme des créatures naturelles, elle doit y arriver ou être détruite, soit du fait des situations nouvelles, soit par un autre être qui se sera adapté lui-même d'une façon plus appropriée. Dans ce problème qui se dresse face à notre Armée de Terre, deux choses dominent : le temps nous presse et nous ne devons pas échouer $»^{33}$.

Texte $n^{\circ} 2$ :

$[b] \ll$ Le Communisme n'est pas en Malaisie un mouvement indigène. C'est l'infection d'une petite fraction de la communauté chinoise par le viruscommuniste transmis par le Bureau du Parti Communiste en Chine. Le Parti Communiste Malais (MCP) a été formé en 1930, et a consacré ses ressources à pénétrer et diriger le monde du travail $»^{34}$.

[c] « Une guerre froide ne peut pas se gagner en fusillant ou en coffrant une population. L'action militaire doit restaurer la loi et l'ordre, mais parallèlement à la lutte militaire, doivent progresser les recherches et l'application del'antidote politique. Citation du Sous- 
secrétaire d'Etat adjoint, Robert D. Murphy: Les guerres froides demandent des nerfs froids. Naturellement, il est important de ne pas sous-estimer son ennemi $»^{35}$.

Des représentations organicistes et vitalistes de l'armée

En ce qui concerne l'extrait [a], l'emploi de la métaphore couvre des représentations organicistes et vitalistes de l'armée. L'affirmation selon laquelle l'Armée est une chose vivanteconditionne l'ensemble des emplois métaphoriques du texte, mais aussi du document en général. Sur le plan purement rhétorique et formel, cette métaphore affirmative repose sur l'analogie établie entre le terme créatureintroduit par le joncteur comparatif comme[8] et l'institution militaire.

Cette analogie induit une dimension dramatique aux «prescriptions » doctrinales du locuteur. Vie ou mort de l'institution, tel est le dilemme posé pour affirmer l'urgence de l'exploitation de la révolution, c'est-à-dire de l'urgence de l'intégration de techniques nouvelles dans le cadre militaire et en particulier dans l'Armée de terre.

Fort de la présence de cette analogie qui fixe le contexte dans lequel émergent les causes premières de la modernisation - du pourquoi il faut faire la révolution-, le locuteur peut légitimement donner un contenu vitaliste et agressif à ses métaphores.

Celles-ci mettent l'accent sur la compétition naturelle entre les corps vivants que sont les Armées : dans la lutte pour la vie, il s'agit de s'adapter ou de mourir non par pur dépérissement, mais en laissant la place à un vainqueur, à un autre être [10]. Il est intéressant de constater que cette métaphore peut aussi bien s'appliquer au phénomène guerrier - l'autre être désignant alors l'ennemi -, que désigner une armée ayant fait sa propre révolution doctrinale dans le cadre d'une compétition existant dans un même camp.

Métaphorisations liées au syntagme guerre froide

Les métaphores des extraits [b] et [c], assurent l'intersection entre l'ensemble des différents réseaux métaphoriques; l'essentiel résidant dans la détermination de la guerre froide comme véritable guerre et comme condition objective de développement d'une compétence professionnelle dont nous avons vu les grandes lignes induisant des représentations de nature différente : le premier de ces réseaux s'articule l'emploi du terme guerre(s); tandis que le second concerne les différentes conditions d'emploi de l'adjectif froid(es).

1. Le mot guerre(s) institue la dimension réelle, non métaphorique, des extraits choisis. Il sert de base objective pour signifier la réalité militaire du conflit; il désigne une réalité palpable, et permet de la sorte de donner une dimension réaliste et pratique à l'expression guerre froide qui, malgré sa déviance par rapport au sens commun, acquiert ainsi de la vraisemblance. Des expressions métaphoriques qui gravitent autour d'elle y trouvent une large légitimité. Elle leur attribue également une certaine force dramatique : c'est bien de guerre(s)dont il s'agit, et non pas d'un simple rapport de force international entre l'Est et l'Ouest ou d'une quelconque forme mineure de faire la guerre.

Le pluriel mis au substantif guerre permet au locuteur de généraliser la portée de ses propositions au-delà d'une expression à l'unique dimension contextuelle : la guérilla malaise n'a été ni la première, ni ne sera sans doute la dernière de ces guerres froides. Dans ce cadre énonciatif qui ouvre la porte à une forme de casuistique, la modélisation de la pratique britannique a la force de l'exemple. Elle permet également d'assurer la 
démarche des autorités de l'ESG dans leur affirmation de la réalité militaire de la guerre froide, tout en faisant de la guérilla sa dimension guerrière spécifique.

2. L'adjectif froide(s)joue le rôle de véritable point d'intersection entre les différents domaines du texte. Il institue le lien entre les référents concernés par la métaphore médicale et organiciste - en tant qu'épithète de nerfs - et le domaine de la réalité - les guerres froides- guerre(s) en général ou cette forme particulière de la guerre qu'est la guérilla. Il permet également d'établir une articulation entre le comportement de l'individu et ce qui concerne le contexte dans lequel le soldat est immergé.

Sur le plan rhétorique, à la différence de l'emploi de la métaphore, - in praesentia ou in abstentia -, il semblerait que nous soyons en présence d'une expression métaphorique per substitutio, qui réaliserait la requalification d'un terme par un autre. La métaphore est per substitutio car elle s'appuie en fait sur une métaphore classique, celle du comportement totalement maîtrisé en période de crise : les nerfs froids, adaptation de l'expression « tête froide ».

Les métaphores relatives à la relation ami-ennemi et à l'action anti-guérilla

Le domaine de la métaphore dans les extraits [b] et [c]concerne deux référents: l'ennemi et les différents aspects de l'action britannique, tant sur le plan du soldat que sur le plan institutionnel et technique.

1. Dans l'extrait [b] le locuteur tente d'induire le manque de légitimité interne de l'ennemi en montrant son hétérogénéité essentielle par rapport à la réalité malaise et, surtout, la dimension artificielle de la situation "pathologique " que cet ennemi exogène crée. Le locuteur insiste sur le fait que celui-ci est lié au PC chinois, et qu'il n'en serait qu'une émanation. Cette illégitimité nationale serait de plus redoublée sur le plan de sa représentativité ethnique : il est minoritaire dans la population malaise du fait de ses origines chinoises.

La métaphore du viruss'insère dans ce cadre énonciatif de dévalorisation de l'ennemi. Non seulement elle le désigne sous une forme minuscule, virulente et pathogène, mais elle permet également, sur le plan discursif, de donner son sens plein à la métaphore qui précède: celle de l'infection pour désigner la présence d'une structure d'origine étrangère - le PC malais - dans un « corps ", ici la communauté chinoise malaise.

De plus, le terme virus renforce le sens du verbepénétrer pour ce qui a trait à la présence de l'ennemi « pathogène » dans le monde du travail. Mais cette fois-ci c'est sa légitimité à la direction des conflits sociaux, politiques et militaires qui est stigmatisée. On remarquera que l'emploi de ce type de métaphores renvoie à une conception générale $\mathrm{du}$ conflit social qui devient illégitime à partir du moment où il se dote d'une organisation autonome forte et qu'il s'écarte d'une expression non "naturelle », voire même spontanée de l'action sociale ou politique. L'ennemi est bel et bien l'organisation de l'adversaire. Elle se confond avec lui.<//r2r:section1>

2. Dans ce contexte, l'extrait [c] fait émerger l'emploi de la métaphore pour désigner les différents modes d'action britanniques préconisés par le général Brazer-Creagh. Les syntagmes antidote politiqueet nerfs froids trouvent leur plein emploi en étant mis en relation, non seulement avec le domaine métaphorique propre à l'extrait antérieur, mais aussi par rapport à celui du texte $n^{\circ} 1$.

L'expression métaphorique antidote politique concerne fondamentalement la capacité de réaction par rapport à l'ennemi : le virus. Cette expression désigne également cette connaissance nouvelle du soldat : l'action, ou plutôt l'agissement politique. 
Cependant, le terme antidotedésigne également un contrepoison de nature défensive. C'est un produit spécifiquement orienté vers la destruction d'un agent pathogène spécifique et auquel cet antidote correspond. La relation Ami-Ennemi demande de l'équilibre ; c'est-à-dire, jamais de surestimation ni de sous-estimation de l'adversaire. Singulièrement c'est la représentation organiciste de la société qui est renforcée. La société apparaît effectivement comme un corps dans lequel va se livrer une bataille entre le virus et l'antidote politique. La désignation métaphorique de mesures militaropolitiques que le locuteur a abondamment décrites permet de la sorte de les présenter sous un aspect synthétique; et de justifier l'amenuisement de la séparation entre l'action politique et l'action militaire.

La présence de cette image, de cette représentation volontariste et positive de l'action politique militairement orientée, assure également la légitimité de cette capacité nouvelle de l'officier. Cette nouveauté d'un savoir-faire politique du militaire est affirmée à couvert sous l'expression métaphorique: le militaire doit avoir des nerfs froids. Il doit posséder les compétences professionnelles nécessaires et suffisantes pour ne pas perdre le contrôle de lui-même face à l'ennemi ; mais aussi pour ne pas se laisser troubler par la dureté de cette forme de conflit. Cette capacité de calme et de maitrise des sentiments est ce qui est requis sur le plan comportemental lorsqu'il s'agit de mener une guerre tout aussi froide ;c'est-à-dire de manier les appareils de coaction, de coercition de masse et de renseignement.

Sur le plan rhétorique, il apparait que la métaphore des nerfs froidsassoitaussi l'emploi spécifique de l'expression, au sens quelque peu modifié,de guerre froidesur laquelle le locuteur se base pour argumenter en faveur des pratiques de la contre-guérilla.

De façon synthétique, on relèvera, par rapport à l'ensemble du document, que l'on ne peut s'empêcher de généraliser cette caractéristique comportementale à laquelle s'ajoute la compétence politique à l'ensemble de l'armée. La jonction des deux réseaux métaphoriques fait ressortir la dimension individuelle du courage et la maitrise physique de soi qu'impose l'action coactive. Cette jonction place en situation de complémentarité le système nerveux d'un homme parfaitement et professionnellement entraîné, avec celui du système hiérarchique et organisé de l'institution militaire, ellemême présentée globalement comme un organisme vivant [a].

Ce réseau métaphorique qui parcourt les deux textes est donc lourd de sens multiples. D'un côté, il assure des représentations modernisatrices de l'Armée en tant qu'institution et, de l'autre, il sert de socle rhétorique à la présentation d'un savoirfaire militaro-politique, tant sur le plan du comportement d'officiers spécialisés, que sur sa possible traduction «organique» de la contre-guérilla comme technique organisationnelle.

Ce spécialiste devra posséder des capacités politiques, des nerfs froids, l'expérience de la guérilla et la pratique des organisations de coaction et de coercition de masse. Il semble que se soient là des indications qui peuvent permettre de définir les principaux critères de formation pour orienter les futurs officiers français dans les Etat-Majors et dans les hiérarchies particulières propres aux guerres froides. 


\section{NOTES}

1. . Voir, pour information sur le concept, P. .Paret, French revolutionary warfare from Indochina to Algeria, New York, Washington, Londres, F.A. Praeger, 1964.

2. . R. Girardet, « Reflexions critiques sur la doctrine militaire française de la guerre subversive ", Revue des travaux de l'Académie des sciences morales et politiques, 1er semestre 1960, p. 232.

3. . Voir, Général Beaufre, Stratégie de l'action, Paris, A.Colin, 1966.

4. . Nous soulignons dorénavant tout trait caractéristique porteur de MMC.

5. . Commandement en chef en Extrême-Orient, Enseignements de la guerre d'Indochine, fasc. II, 1955, p. 47.

6. . Général Beaufre, Stratégie de l'action, op. cit., 1966.

7. . G. Périès, « Doctrine de la sécurité nationale et national-catholicisme, deux sources théoriques du Processus de réorganisation nationale en Argentine (1976-1983) », DEA, Université de Paris I, 1986, p. 112.

8. . E. Vásquez, PRN-La última, origen, apogeo y caída de la dictadura militar, Buenos Aires, Eudeba, 1985, p. 326.

9. . Le thème de cette première partie $a$ fait l'objet d'une publication antérieure, $G$. Périès, La métaphore médico-chirurgicale dans le discours militaire de la " guerre révolutionnaire ", Mots, les langages du politique, n² 26, PFNSP/CNRS/ENS, mars 1991, p. 5.

10. . C. Mauron, Des métaphores obsédantes au mythe personnel. Introduction à la psychocritique, Paris, José Corti, 1962, p. 35 et suiv.

11. . Anonyme, « Guerre révolutionnaire et médecine moderne », Revue Militaire d'Information, $\mathrm{n}^{\circ} 283,1957$, p. 47.

12. . Débat entre les commandants Hogard, Cogniet et Renaudin, « La guerre révolutionnaire, tactiques révolutionnaires et contre-révolutionnaires », Ecole Supérieure de Guerre, 18 juin 1957.

13. . C. Perelmann, L. Olbrechts-Tyteca, Traité de l'argumentation, Bruxelles, Editions de l'Université de Bruxelles, 1988, p. 729.

14. . Ibid., p. 535.

15. . Nous employons « sème » comme « trait minimum porteur de sens ».

16. . Général Beaufre, Introduction à la stratégie, Paris, A. Colin, 1965, p. 116.

17. . H.Morier, Dictionnaire de poétique et de rhétorique, Paris, PUF, 1989, p. 687.

18. . Capitaine A. Argoud, «La guerre psychologique », Revue de Défense Nationale, mars 1948, p. 291 et avril 1948, p. 460.

19. . Colonel J. Defrasne, « Caractéristiques d'emploi de l'arme psychologique », Revue militaire générale, novembre 1957, p. 520.

20. . C. Perelmann, L.Olbrechts-Tyteca, op. cit., p. 535.

21. . Général Artola, ¡Subversión !, Lima, Editorial Jurídica, 1976, pp. 10-11.

22. . Au Pérou, en 1965.

23. . H.Morier, Dictionnaire de poétique et de rhétorique, Paris, PUF, 1989, p. 679

24. . Commandement en chef en Extrême-Orient, Enseignement..., op. cit., p. 46.

25. . Anonyme, « Guerre révolutionnaire... », op. cit., p. 47.

26. . Voir, en particulier, la RMI, n 281 , février-mars 1957, consacrée à : « La guerre révolutionnaire, données et aspects, méthodes de raisonnement, parade et riposte ». 
27. . G. Périès, « L'Arabe, le Musulman, l'Ennemi dans le discours militaire de la guerre révolutionnaire pendant la guerre d'Algérie », Mots, Les langages du politique, $\mathrm{n}^{\circ} 30$, PFNSP/CNRS/ENS, mars 1992, p. 53.

28. . C. Perelmann, L. Olbrechts Tyteca, op. cit., p. 542.

29. . Voir, à ce propos, la médicalisation des analogies dans ce contexte : « Ainsi le voulait notre métier. Pouvait-il en être autrement? Nous étions devenus pareils aux chirurgiens habitués aux pires plaies des accidentés de la route, qui entrent en sifflant dans la salle d'opération, amputent le blessé et repartent sereinement ». J.-P.Vittori, Confessions d'un professionnel de la torture, Paris, Ramsay, 1980, pp. 12-13.

30. . G. Périès, « Conditions d'emploi des mots interrogatoire et torture dans le discours militaire pendant la guerre d'Algérie ", Mots, les langages du politique, n 51 , PFNSP/ CNRS/ENS juin 1997, p. 41.

31. . Texte paru en anglais sous le titre : Com J.H Cushman, " Harness the Revolution », Military Review, janvier 1955.

32. . Texte paru en anglais sous le titre : Gal Brazer-Creagh, "Waging a cold war ", Journal of Royal United Service Institution, mai 1954.

33. . Commandant J. H. Cushman, op cit, p. 11.

34. Général Brazer-Creagh, op cit, p. 1.

35. . Général Brazer-Creagh, op. cit, p. 11.

INDEX

Index chronologique : guerre froide

Mots-clés : contre-insurrection, Doctrine militaire, ennemi, Militaires, question coloniale, sécurité intérieure

Index géographique : Algérie 